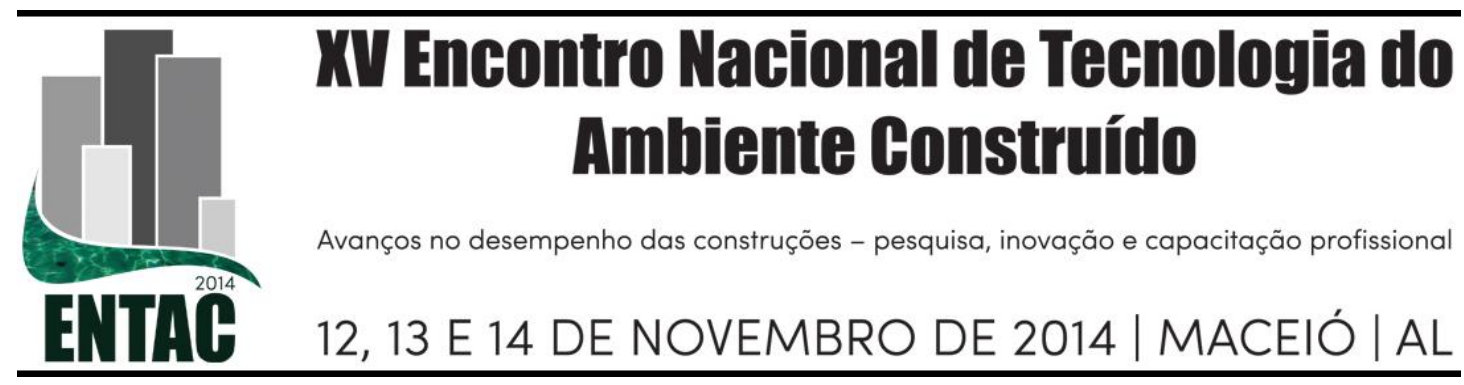

\title{
SISTEMA PUXADO DE PLANEJAMENTO E CONTROLE DA PRODUÇÃO
}

\author{
BONI, Adriana Cristina (1); PALIARI, José Carlos (2); SERRA, Sheyla Mara \\ Baptista (3)
}

Núcleo de Pesquisa em Racionalização e Desempenho de Edificações (NUPRE), Programa de PósGraduação em Estruturas e Construção Civil (PPGECIV), Universidade Federal de São Carlos (UFSCar)

(1) Doutoranda, adrianacristinaboni@yahoo.com (2) Professor, jpaliari@ufscar.br (3) Professora, sheylabs@ufscar.br

\begin{abstract}
RESUMO
Na indústria da construção civil continuamente são desenvolvidas pesquisas nos âmbitos da Construção Enxuta e do Planejamento e Controle da Produção (PCP), visando identificar formas mais racionalizadas de gestão da construção. Um dos principais aspectos abordados nesta área temática é compreender quando um sistema de PCP deve passar de empurrado a puxado, agregando maior previsibilidade e confiabilidade ao processo. Também se torna importante identificar quais os principais parâmetros e métricas de acompanhamento do PCP. Observou-se que existem diferentes referências que apresentam abordagens distintas de planejamento na construção a partir do entendimento do Projeto do Sistema da Produção (PSP). Assim, é objetivo deste trabalho realizar uma discussão teórica sobre o uso combinado das técnicas tradicionais de PCP com as da mentalidade enxuta para que se obtenha um sistema de fluxo contínuo e puxado de produção. As principais abordagens focam nos procedimentos do Pull Planning e do Takt Time Planning (TTP) que podem ser vinculados ao Last Planner System (LPS). Como resultado preliminar deste artigo, é gerada uma hipótese de integração dos sistemas por meio da integração das fases do PSP e do LPS, que ainda necessita ser testada.
\end{abstract}

Palavras-chave: Construção enxuta, Planejamento e controle da produção, Last Planner, Sistema de planejamento puxado, Planejamento tempo takt.

\begin{abstract}
In the civil construction industry researches constantly are developed in the areas of Lean Construction and of Production Planning and Control (PPC), to identify more rationalized forms of construction management. One of the main issues approached in this thematic area is to understand when a PPC system should pass from pushed to pulled, adding greater reliability and predictability to the process. It also becomes important to identify the key parameters and metrics for monitoring the PPC. It was observed that there are different references that present different planning approaches in building from an understanding of the Project Production System (PPS). Thus, the aim of this work is to realize a theoretical discussion of the combined use of traditional techniques of PPC with those of lean thinking in order to obtain a continuous flow and pulled PPC. The main approaches focus on the procedures of Pull Planning and TTP that can be linked to the Last Planner System (LPS). As a preliminary result of this article a hypothesis of integration of the systems is generated by the integration of PPS and LPS phases, which still needs to be tested.
\end{abstract}

Keywords: Lean Construction, Planning and production control, Last Planner, Pull planning system, Takt time planning. 


\section{INTRODUÇÃO}

O planejamento tradicional na construção civil é, muitas vezes, direcionado para o desempenho global do empreendimento, não se preocupando com análises específicas de cada unidade produtiva. Como efeito, surgem deficiências relacionadas à utilização de recursos e cumprimentos das metas planejadas que ocorrem em função da incerteza e da variabilidade que são negligenciadas ao longo do processo. Estudos recentes revelam que o uso combinado do Planejamento e Controle da Produção (PCP) com técnicas mais avançadas de planejamento, como o Pull Planning e o Takt Time Planning (TTP), pode melhorar a programação de atividades, aumentando a confiabilidade e o fluxo contínuo de operações, reduzindo o tempo de ciclo de cada atividade e a duração total de projeto. O estudo e comparação de diferentes abordagens do PCP se torna importante para obter mais conhecimento sobre o cenário de planejamento e controle a ser adotado.

O objetivo deste trabalho é discutir sobre alguns procedimentos de planejamento na construção civil que apresentam potencial de melhorias para que se obtenha um sistema de fluxo contínuo e puxado de PCP.

\subsection{Método da pesquisa}

Utilizou-se a pesquisa bibliográfica como estratégia de investigação, sendo que a mesma está delimitada às seguintes áreas temáticas: Projeto e Controle do Sistema de Produção e Planejamento e Controle da Produção (Figura 1), sendo identificados cinco principais autores. A pesquisa bibliográfica é parte de um estudo exploratório e possui a vantagem de examinar com maior profundidade as diferentes opiniões e resultados científicos sobre os dados requeridos pelo problema de pesquisa que se encontram dispersos em várias literaturas (GIL, 2012).

\section{Figura 1 - Método da pesquisa}

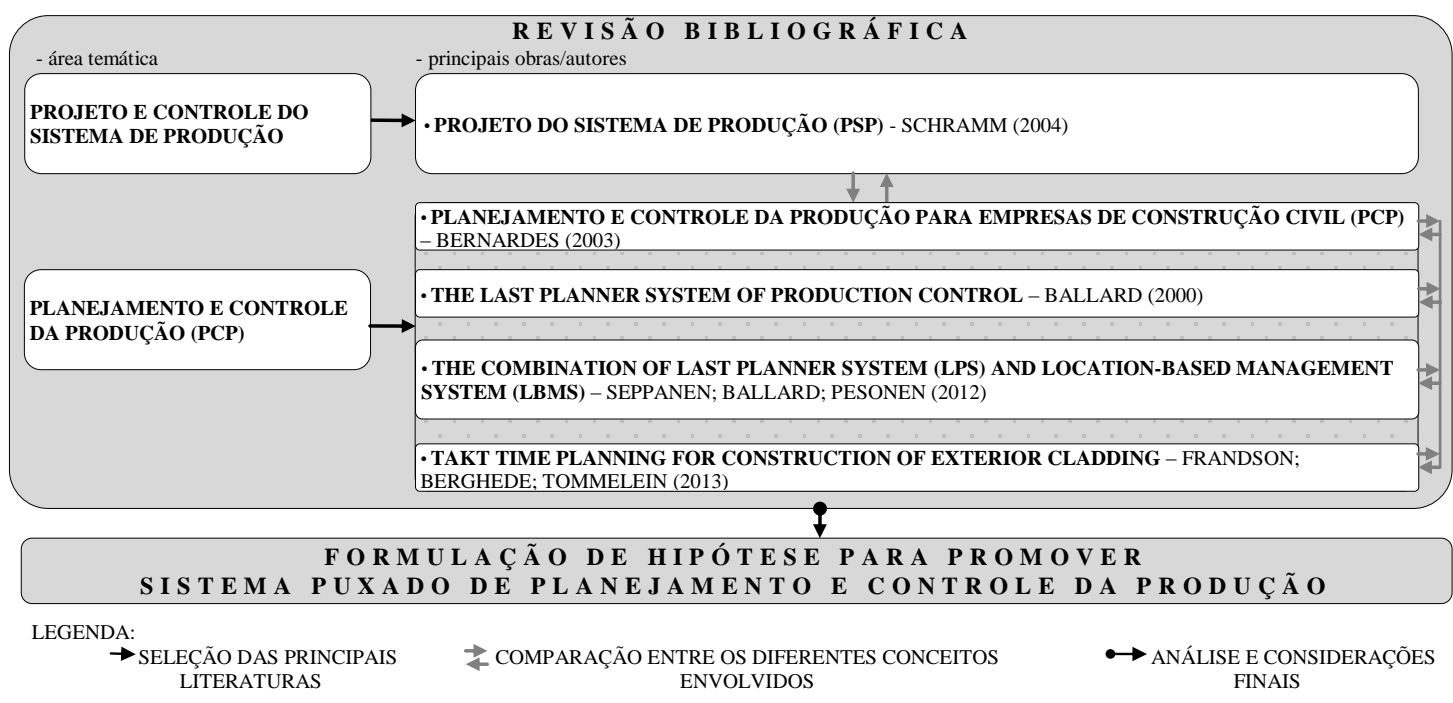

\section{PROJETO E CONTROLE DO SISTEMA DE PRODUÇÃO}

Entende-se que a análise do sistema de produção permite discutir e definir a estratégia de produção desejada. Assim, por meio da compreensão do sistema de produção é possível definir o conjunto de decisões necessárias para aumentar as oportunidades de melhoria no processo de PCP. Neste item é definido o que é um sistema de produção e qual a importância do projeto do sistema de produção no contexto do planejamento. 


\subsection{Sistema de produção}

Os elementos de um sistema reúnem, de forma coordenada e lógica, ideias, princípios, técnicas e ferramentas para a consecução de um objetivo global de uma determinada área do conhecimento (CHURCHMAN, 1972). Neste sentido, pode-se afirmar que um sistema de produção é a reunião lógica dos elementos necessários para a produção de um produto ou um serviço.

$\mathrm{Na}$ indústria da construção civil, o sistema de produção pode ser entendido como uma estrutura que envolve os projetos do produto e o projeto do sistema de produção (design and making) (SCHRAMM, 2004). O projeto do produto influencia diretamente no custo de produção bem como no desempenho do sistema de produção, colaborando para definir estratégias de PCP. O sistema de produção apresenta, portanto, duas interfaces: uma com o processo de projeto do produto e outra com o processo de PCP. Na interface do sistema de produção com o PCP é possível aumentar as oportunidades de se reduzir parcelas de atividades que não agregam valor (transporte, espera e inspeção) caracterizando um sistema puxado de planejamento e de produção. Um sistema pode ser considerado puxado quando pode ser previsto com maior precisão dentro de uma janela de confiabilidade e pode ser considerado contínuo quando existem buffers de pequenas durações entre as atividades consecutivas das equipes (BALLARD, 2000; BALLARD et al., 2001; KOSKELA, 2000; SCHRAMM, 2004).

Segundo Ballard et al. (2001), a teoria do sistema de produção proposta por Koskela (2000) deve ser o ponto de partida para a construção civil, pois as desenvolvidas para a manufatura, como o Sistema Toyota de Produção, não são adaptáveis às situações de produção existentes neste setor. Na teoria de Koskela (2000), as diretrizes estabelecidas buscam cumprir três objetivos principais: executar a tarefa (task), minimizar o desperdício (flow) e maximizar o valor (value). Para a consecução desses objetivos, Ballard et al. (2001) propõem considerar o sistema de produção como entrada do PCP para a divisão do trabalho a ser realizado, ou seja, considerar no planejamento tradicional da Estrutura Analítica do Projeto (EAP) o conceito de Tarefa/Fluxo/Valor (TFV) (Task / Flow/Value) para a divisão de tarefas.

\subsection{Projeto do sistema de produção (PSP)}

O trabalho principal pesquisado para a discussão neste item foi desenvolvido pelo autor Schramm (2004), que fez a proposição das etapas necessárias para a elaboração do PSP para empreendimentos habitacionais de interesse social. Para esse autor, o objetivo do PSP é compreender o processo de análise e discussão de alternativas para o sistema de produção dos empreendimentos e criar condições básicas para a primeira forma de PCP, atenuando a variabilidade e a incerteza, inerentes dos processos produtivos na construção. A maioria dos recursos empregados no sistema de produção é definida durante a fase do PSP. Assim, reduzindo-se a variabilidade por meio da estabilização da produção obtida com o PSP é possível buscar a manutenção de um fluxo contínuo dos recursos de uma unidade-base de produção para a outra. A unidade-base de produção é considerada como qualquer unidade repetitiva representada por um pavimento, um apartamento, uma casa ou um sobrado, podendo ser dividida em zonas de trabalho. Para as zonas de trabalho, além da definição dos recursos, o PSP planeja definindo quem deve estar envolvido em qual papel para decisões a respeito de como o trabalho físico deverá ser realizado (BALLARD et al., 2001; SCHRAMM, 2004).

As principais métricas para acompanhamento do PSP são tempo de ciclo e indicadores de produtividade, possuindo como parâmetro de demanda o tempo takt. Para Schramm 
(2004), a concepção do sistema de produção inicia-se pela definição do tempo takt, que dita o ritmo de produção sincronizado ao ritmo de vendas (demanda). A finalidade do tempo takt passa ser a de sincronizar as operações nas zonas de trabalho estabelecendo um ritmo para a produção, nivelando a capacidade produtiva. O tempo takt será mais discutido no item 3.4. Na primeira etapa do PSP, deve-se fazer a definição da sequência tecnológica da obra e o pré-dimensionamento da capacidade de recursos; as taxas de produtividade das equipes refletem diretamente em um ritmo de produção que pode ser estabelecido. Com base nas informações geradas nesta etapa, também é possível estimar o tempo de conclusão de uma unidade-base. Na etapa de definição de estratégia de execução do empreendimento busca-se realizar sua divisão em zonas de trabalho, criando pequenos lotes de produção; os lotes podem ser executados de forma sequencial ou em paralelo e, para os mesmos, são dimensionadas e alocadas as equipes para uma situação ideal de trabalho onde as atividades são executadas em fluxo contínuo e ritmo pré-estabelecido. Para a manutenção do prazo final de execução podem ser necessários estudos com relação à sincronização entre alguns processos específicos, denominados de processos críticos, pois podem vir a limitar a capacidade do fluxo de trabalho como um todo (SCHRAMM, 2004).

O método para elaboração do PSP proposto por Schramm (2004) abrange as seguintes etapas: definição da sequência de execução e pré-dimensionamento da capacidade dos recursos de produção para a unidade-base; estudo dos fluxos de trabalho para a unidadebase; definição da estratégia de execução do empreendimento; estudo dos fluxos de trabalho no empreendimento; dimensionamento da capacidade dos recursos de produção para a consecução do empreendimento como um todo e identificação e projeto de processos críticos no empreendimento (conforme Figura 2).

Figura 2 - Etapas do PSP para empreendimentos habitacionais

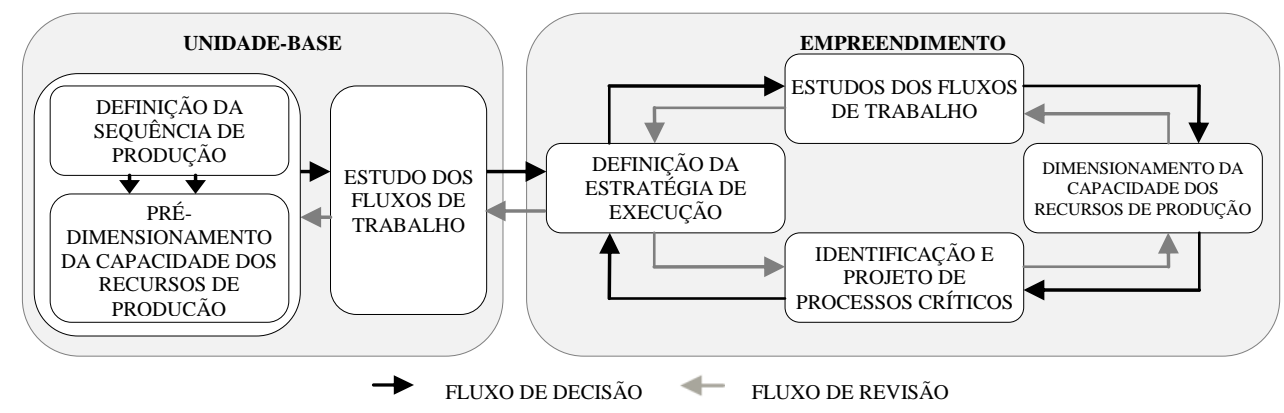

Fonte: Schramm (2004)

De um modo geral, o PSP tem foco no estudo do fluxo de trabalho. O potencial de melhoria oferecido pelo PSP é fornecer informações de entrada para a elaboração dos planejamentos de longo, médio e curto prazo, auxiliando no atendimento ao plano de ataque, à sincronização de operações e manutenção dos ritmos dos processos produtivos.

\section{PLANEJAMENTO E CONTROLE DA PRODUÇÃO}

\subsection{Planejamento tradicional}

$\mathrm{Na}$ construção civil, de um modo geral, as formas tradicionais de planejamento tem se resumido à geração de orçamentos e planos de longo prazo direcionados ao empreendimento. As informações utilizadas para a geração do planejamento de longo 
prazo são baseadas normalmente na experiência obtida em empreendimentos anteriores, entendido como planejamento empurrado. Neste caso, o uso da informação para a execução no curto prazo ocorre somente de forma verbal, sem vínculos com os níveis de planejamento de médio e curto prazo, que geralmente são inexistentes. Desta forma, a incerteza e a variabilidade, que são inerentes do processo produtivo, são negligenciadas. Como efeito, há utilização ineficiente de recursos e significativo desvio das metas planejadas em relação às executadas, onde o controle e a realização de ações corretivas no sistema de produção são mais difíceis ou até ineficientes (BERNARDES, 2003).

Para o acompanhamento da produção normalmente são utilizadas métricas ou parâmetros de controle dentre os vários existentes, como desvios de prazo e as curvas $\mathrm{S}$ para análise do avanço físico da produção (OLIVEIRA, 2009).

O planejamento tradicional não possui foco nos fluxos de trabalho, demandando melhorias para o planejamento da manutenção dos ritmos e sincronização das atividades.

\subsection{Last Planner System (LPS) de controle de produção}

Este item teve como abordagem principal a tese de Ballard (2000) que teve por objetivo desenvolver um sistema de planejamento puxado para a produção com foco na função controle. O Last Planner System (LPS) é um sistema puxado de controle da produção, projetado para a construção civil, com foco no fluxo da produção que permite que as atividades iniciem apenas quando todas as restrições forem removidas. Os integrantes do grupo que fazem a programação das atividades para a produção são denominados de "os últimos planejadores" (the Last Planners) (BALLARD, 2000).

A métrica-chave do LPS é a Porcentagem de Planos Concluídos (PPC) que avalia a quantidade das tarefas concluídas e enfatiza a melhoria da produtividade das equipes (crew's PPC) (BALLARD, 2000).

O método para implementação do LPS ocorre por meio de cinco elementos: master schedule; lookahead process; phase schedule (pull planning); weekly work plans e PPC. A programação de longo prazo (master scheduling) gera as datas marco e as principais atividades com suas respectivas durações; nesta fase os dados sobre duração e recursos são informações "empurradas" para o sistema de produção. A partir da programação de longo prazo ocorrem seleção, sequenciamento e dimensionamento do trabalho que pode ser feito para processo seguinte, o lookahead. O sistema passa a ser puxado por meio do processo lookahead, pois o que "deveria ser feito" passa a ser "o que deve ser feito". No processo lookahead são selecionadas as tarefas em potencial para serem realizadas em uma janela de três a doze semanas, observando que o número de semanas varia de acordo com as características do empreendimento.

Para controlar as atividades, Melles e Wamelink (1993) citados por Ballard (2000), sugerem a janela de seis semanas - o six weeks scheme. Na sequência é realizado o planejamento por fases (phase schedule ou phase pull planning) que tem por objetivo coordenar as ações para um período maior que as janelas fixadas no processo lookahead. Nesta fase, o planejamento atende as demandas das tarefas a jusante, onde são especificados os principais conflitos operacionais e as atividades onde ocorrerá a transferência do trabalho (handoffs) de uma unidade-base de produção para a próxima (conforme Figura 3).

A técnica empregada no pull planning consiste em programar cada fase da produção, de traz para frente, a partir de uma data marco, contando com a colaboração dos principais 
envolvidos na produção; a ideia é garantir que seja realizada a melhor programação para as equipes (incluindo a participação dos fornecedores). Nesta fase, pode se obter maior transparência do processo por meio do emprego de ferramentas visuais.

Figura 3 - Detalhamento de cada atividade (ferramenta visual)

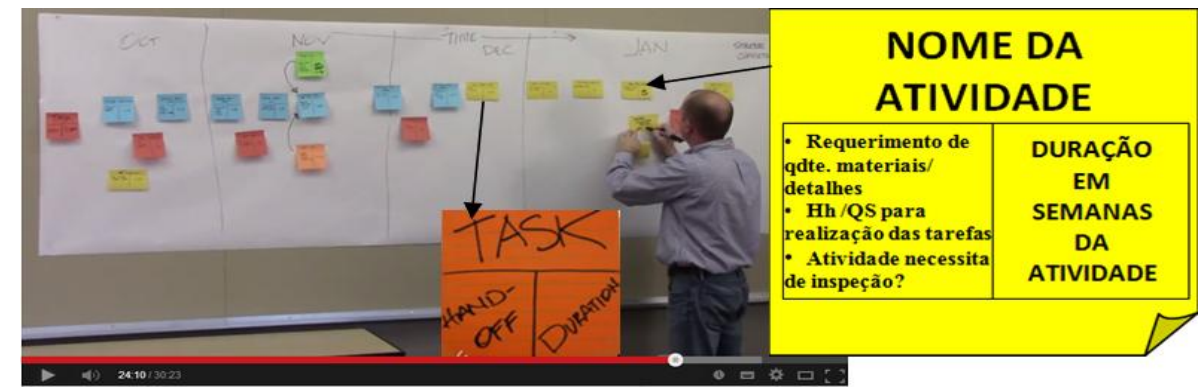

Fonte: Pull planning (2013)

Um exemplo de ferramenta visual que pode ser empregada no pull planning é o indicado na Figura 3: notas adesivas, contendo todas as informações necessárias para a execução de uma tarefa, são fixadas em quadros no local onde são realizadas as reuniões com os envolvidos na produção. O próximo passo é o planejamento semanal (WWP) que transforma as tarefas que "podem ser feitas" na certeza de que "irão ser feitas". O WWP é programado com a colaboração da equipe de produção e promove maior comprometimento entre envolvidos na produção e gerando maior confiabilidade na execução dos serviços. Por fim, o PPC, razão entre as atividades executadas e as planejadas, indica o desempenho de cada equipe em cada semana e permiti discutir a razão da não conclusão das tarefas do período (BALLARD, 2000).

O LPS possui foco no controle do fluxo da produção associado aos planejamentos de médio e curto prazo. O potencial de melhoria oferecido pelo sistema é buscar um planejamento puxado por meio de um processo colaborativo, com foco na função controle; o LPS permite a combinação com outros sistemas de controle da produção.

\subsection{Uso combinado do LPS com o Location-Based Management System (LBMS)}

O presente item tem como referencial teórico o trabalho dos autores Seppänen, Ballard e Pesonen (2010) que apresenta como estudo de caso a experiência da empresa de construção Skanska Finland's, cujo objetivo foi analisar o emprego do uso combinado do LBMS com o LPS na fase de pré-planejamento da produção.

As principais métricas dos sistemas analisados são os índices de produtividade e o PPC. O LBMS é essencialmente um sistema técnico, baseado em dados de produtividade para fornecer informações durante as tomadas de decisões ao longo do PCP. Assim, o LBMS, que no passado utilizava somente a técnica das linhas de balanço (LB) e o do CPM, evoluiu demandando que quantitativos de materiais, equipamentos e dimensionamento de equipes de produção fossem endereçados a cada lote de produção. Neste contexto, surgiu o conceito sobre "Location Breakdown Structure (LBS)" que é a readequação da Estrutura Analítica de Projetos (EAP) para cada unidade de produção, sendo que estas unidades não são necessariamente repetitivas.

O método empregado foi o planejamento baseado no local de produção por meio da readequação da EAP para cada unidade-base de produção (lotes). O processo do LBMS é iniciado pela divisão das zonas de trabalho no projeto. É uma fase de préplanejamento que gera os planos para as unidades de produção (location-based plan). 
As definições das unidades de trabalhos são hierárquicas e definidas conforme a sequência de produção do LBS. No plano é definido explicitamente todo o trabalho que deverá ser realizado para conclusão de um lote (unidade-base de produção); somente depois a equipe de produção poderá mover-se para a próxima unidade. A quantificação de trabalho por local é o ponto de partida para o location-based plan. O dimensionamento da equipe é realizado conforme os índices de produtividade, ou seja, a duração de cada atividade (h) é calculada dividindo-se a quantidade de serviço (QS) em cada unidade de produção pelo consumo de trabalho - número de homens $(\mathrm{H})$ por unidade, alertando que cada atividade pode conter múltiplos itens a serem quantificados, com diferentes índices de produtividade.

Para o estudo de caso da Skanska Finland's o LBMS foi empregado para analisar a produtividade, tendo por objetivo a redução das durações estimadas no cronograma mestre. As atividades contidas no cronograma mestre (planejamento empurrado) foram alocadas em vinte unidades de produção e as principais operações foram sincronizadas (planejamento puxado). A partir disto, foi realizada a programação por fases (phase schedule) conforme as técnicas do LPS (planejamento puxado), onde os empreiteiros discutiram os detalhes da programação em conjunto com os subcontratados (reunião um). Na reunião um, o projeto foi fixado em um quadro, onde as zonas de trabalho foram delimitadas com canetas coloridas (Figura 4) utilizando-se o LBS; também foi empregado o método com notas adesivas do LPS.

Figura 4 - Proposta de programação por fases com uso da LBS

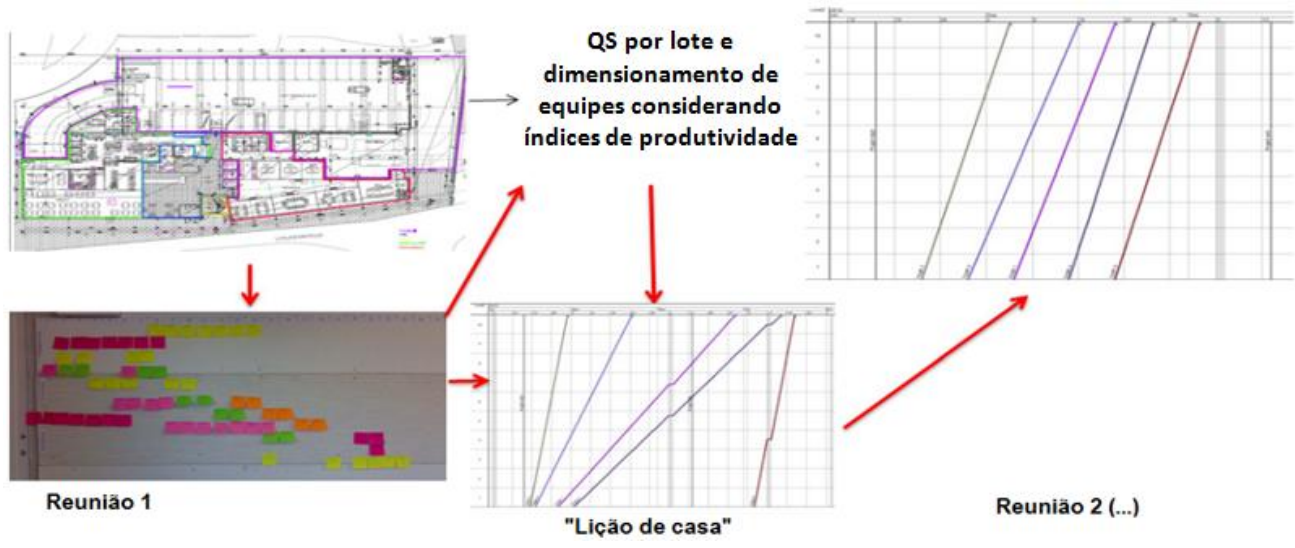

Fonte: Seppänen, Ballard e Pesonen (2010)

O quadro foi dividido verticalmente pelas zonas de trabalho e horizontalmente pelo tempo (obtidos do cronograma mestre) e os tempos restantes foram alocados como buffers entre as atividades. Com locais e durações definidas, cada subcontratado levou os dados para casa onde foram estimados QS e homens-hora por local de execução. Na reunião dois ocorreu o balanceamento do fluxo de trabalho para identificar as operações que possuíam produtividade mais lenta, a fim de aumentar ou diminuir os recursos e alterar a lógica para melhorar a programação de todas as equipes envolvidas no período determinado. No estudo de caso da Skanska Finland's, como resultado do uso combinado do LPS com o LBMS, o tempo de ciclo para a produção de um pavimento passou de cinco dias para quatro dias a partir do terceiro pavimento, aumentando o PPC de $64 \%$ para $78 \%$.

O LBMS possui foco no fluxo ininterrupto das equipes associado ao planejamento do local de produção (planejamentos de médio e curto prazo). O potencial de melhoria dos 
sistemas apresentados se encontra no foco para produtividade e sincronização das atividades nas unidades-base de produção.

\subsection{Takt Time Planning (TTP)}

O presente item tem como referencial teórico o trabalho dos autores Frandson, Berghede e Tommelein (2013) que possui como estudo de caso o centro de saúde de Sacramento, Califórnia. O objetivo do estudo foi analisar os benefícios da programação para a produção de um serviço (fachada exterior do edifício) utilizando o TTP.

Segundo Frandson, Berghede e Tommelein (2013), a principal métrica empregada como parâmetro para a sincronização de operações nas unidades de produção foi o tempo takt, que é o valor a ser calculado e fixado para promover o fluxo contínuo de trabalho (paced work flow).

"Takt" é uma palavra de origem germânica que expressa o ritmo de produção necessário para atender a demanda. Segundo Alvarez e Antunes Jr. (2001), matematicamente, o tempo Takt resulta da razão entre o tempo disponível para a produção e o número de unidades a serem produzidas. Este parâmetro de demanda é largamente empregado no sistema de produção, seja da manufatura, na indústria da construção, ou outros. $\mathrm{O}$ tempo takt pode ser calculado pela razão entre o "tempo disponível para a produção" e o "tempo real demandado pelo cliente"; o cliente pode ser a tarefa à jusante, a equipe seguinte ou a próxima unidade-base de produção. Por meio do tempo takt são obtidas as linhas de ritmo de produção que estabelecem um índice a ser alcançado para verificar se a taxa de demanda do cliente será cumprida e não ultrapassada, sincronizando as operações (tarefas) nas zonas de trabalhos descritas nos itens 2.2 (PSP) e 3.3 (LBMS).

Na Figura 5 de Frandson, Berghede e Tommelein (2013), item (b), o tempo está alocado nas ordenadas; nas abscissas estão alocadas as zonas de trabalho divididas previamente no projeto pela equipe de produção - item (a). Em cada zona de trabalho são estabelecidas as operações (tarefas) a serem realizadas por cada equipe e o tempo demandado para cada uma destas. As linhas de ritmo, definidas pelo tempo takt, são as linhas em vermelho. Se o tempo takt demandado para estabelecer o ritmo de produção for de três dias para a zona 1, por exemplo, todas as operações que estiverem abaixo da linha estão adiantadas e todas as operações que estiverem acima da linha estão atrasadas. Para sincronizar as tarefas e garantir fluxo contínuo nos handoffs entre as operações pode-se, por exemplo, redimensionar a equipe, verificar as causas do não cumprimento das tarefas, entre outros.

\section{Figura 5 - Estudo do tempo takt e durações das atividades nas zonas de trabalho}

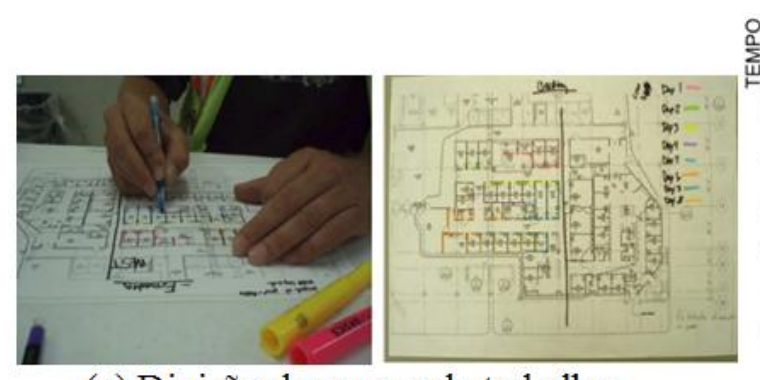

(a) Divisão das zonas de trabalho Fonte: Frandson, Berghede e Tommelein (2013)

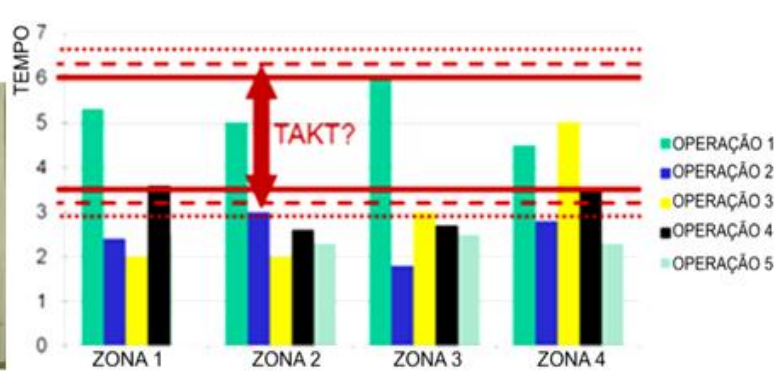

(b) tempo takt

O método abrange a implementação das seis fases do TTP. A fase um compreende a obtenção das informações (gather information); observando operação a operação, 
busca-se identificar qual trabalho deve ser feito e exatamente onde. $\mathrm{Na}$ fase dois ocorre a definição das zonas de trabalho, que na verdade serão zonas de controle. As zonas ideais serão aquelas em que todas as atividades possuírem a mesma duração para serem completadas. O sequenciamento das atividades é realizado na fase três. Para compreender o sequenciamento das atividades existe a necessidade de reuniões para coordenação das equipes, identificando qual equipe irá trabalhar antes ou depois de quem e quantos "passos e partes" cada atividade será completada. Na fase quatro há o balanceamento do fluxo de trabalho, onde são respondidas as seguintes questões: "quais atividades da zona de trabalho precisam ir mais devagar?"; "qual deverá ser a mais rápida?"; "como as zonas podem ser ajustadas para equilibrar o fluxo de trabalho?". Na fase cinco ocorre o estudo da duração individual de cada atividade; equilibrar o fluxo de trabalho requer estudos da primeira execução (protótipo) (first-run studies) para estabelecer durações mais precisas e avaliar as operações em cada zona. Os estudos da primeira execução são fundamentais para verificar a solidez das informações constantes nos detalhes fornecidos em projeto. O balanceamento das atividades não é algo que ocorre de imediato. Pelo contrário, é algo estabelecido de forma gradual por meio da melhoria contínua estabelecida pelo processo do takt time. Por fim, na sexta fase ocorre o cálculo do tempo takt representado anteriormente na Figura 5.

Por meio da implementação do TTP na execução das fachadas leste e oeste do centro de saúde, a duração inicial das atividades estimadas num total de onze meses (cronograma mestre) foi reduzida para cinco meses de execução. O método demandou mais trabalho do que o tradicional, mas ocorreu o aprendizado e cada membro da equipe foi solicitado a pensar sobre as alternativas de sequenciamento e melhores métodos para a execução (FRANDSON; BERGHEDE; TOMMELEIN, 2013).

O TTP possui foco no ritmo do fluxo de trabalho das equipes (planejamento de curto prazo). O potencial de melhoria oferecido pelo sistema é a sincronização e ritmo das unidades de produção obtidas com o emprego do tempo takt.

\section{ANÁLISE E CONSIDERAÇÕES FINAIS}

A questão principal que motivou esta pesquisa foi compreender as características do PCP, as diferenças e similaridades entre os sistemas empurrado e puxado de acordo com as referências selecionadas. Desta forma, a partir dos estudos descritos, foi realizada uma análise comparativa teórica conforme Quadro 1, com base nas métricas e parâmetros utilizados, suas principais características e diferenças e similaridades percebidas na análise realizada.

Observou-se que o PSP é a base de informações de entrada para os PCP empurrado ou puxado, auxiliando no atendimento ao plano de ataque e na manutenção dos ritmos dos processos produtivos. Entretanto, no planejamento tradicional, o mesmo pode não ser levado em consideração, gerando problemas como os mencionados anteriormente. Já os PSP, TTP e LBMS podem ser entendidos como sistemas puxados de produção por apresentar uma estrutura de planejamento coerente com o processo de controle e programação da obra.

A partir das informações obtidas nas referências selecionadas foi possível verificar que o LPS promove melhorias na forma tradicional de concepção do PCP. Contudo, para maior eficiência do LPS ainda é necessário considerar os procedimentos estabelecidos pelos PSP, TTP e LBMS, tais como produtividade, sincronização e manutenção do ritmo de produção nas unidades-base. 


\section{Quadro 1 - Comparação entre os sistemas de PSP e PCP}

\begin{tabular}{|c|c|c|c|}
\hline Sistemas & $\begin{array}{c}\text { Principais } \\
\text { métricas ou } \\
\text { parâmetros dos } \\
\text { sistemas }\end{array}$ & Características & $\begin{array}{c}\text { Diferenças e similaridades entre os } \\
\text { sistemas }\end{array}$ \\
\hline PSP & $\begin{array}{l}\text { Tempo de ciclo; } \\
\text { Tempo takt }\end{array}$ & $\begin{array}{l}\text { Fornece informações de } \\
\text { entrada para os PCP } \\
\text { empurrado ou puxado, } \\
\text { auxiliando no atendimento } \\
\text { ao plano de ataque e na } \\
\text { manutenção dos ritmos dos } \\
\text { processos produtivos }\end{array}$ & $\begin{array}{l}\text { Não é classificado como sistema de } \\
\text { PCP }\end{array}$ \\
\hline $\begin{array}{l}\text { Planejamento } \\
\text { tradicional }\end{array}$ & $\begin{array}{l}\text { Desvios de prazo } \\
\text { e Avanço físico }\end{array}$ & $\begin{array}{l}\text { Planejamento empurrado } \\
\text { com foco no processo global } \\
\text { do empreendimento }\end{array}$ & $\begin{array}{l}\text { Não colabora em análises específicas } \\
\text { para as unidades produtivas }\end{array}$ \\
\hline LPS & PPC & $\begin{array}{l}\text { Planejamento puxado com } \\
\text { foco na função controle }\end{array}$ & $\begin{array}{c}\text { Sistema distinto do PSP, apresentando } \\
\text { integração nos planejamentos de longo, } \\
\text { médio e curto prazo }\end{array}$ \\
\hline LBMS & $\begin{array}{l}\text { Indicador de } \\
\text { produtividade }\end{array}$ & $\begin{array}{l}\text { Sistema de PCP puxado, com } \\
\text { foco na produtividade e } \\
\text { sincronização das atividades }\end{array}$ & \multirow{2}{*}{$\begin{array}{l}\text { Todos são sistemas de PCP puxados e } \\
\text { seguem o escopo do PSP, com foco na } \\
\text { divisão do trabalho em unidades-base } \\
\text { de produção para: estruturação da EAP; } \\
\text { dimensionamento das equipes por } \\
\text { unidade-base de acordo com os índices } \\
\text { de produtividade; sincronização das } \\
\text { operações em cada unidade e entre as } \\
\text { unidades; manutenção de ritmo de } \\
\text { produção e redução do tempo de ciclo } \\
\text { de cada unidade }\end{array}$} \\
\hline TTP & Tempo takt & $\begin{array}{l}\text { Sistema de PCP puxado com } \\
\text { foco na sincronização e no } \\
\text { ritmo de produção }\end{array}$ & \\
\hline
\end{tabular}

Após análise da teoria apresentada sucintamente neste artigo, entendeu-se como provável conceber um sistema de PCP puxado elaborado a partir do plano mestre de produção, combinando o PSP com a primeira fase do LPS, como sugerido na Figura 6.

\section{Figura 6 - Hipótese para promover sistema puxado de PCP}

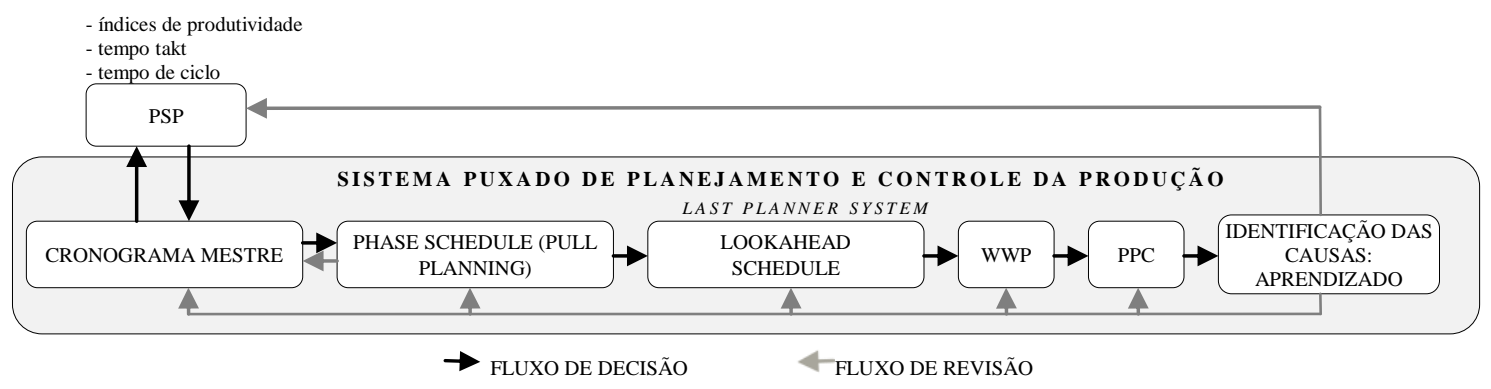

Conforme Figura 6 propõe-se que no início do processo as sequências das atividades e suas durações (cronograma mestre) sejam revisadas por meio de dados mais realísticos, onde o processo não será baseado apenas nas interdependências das atividades, mas também em um fluxo contínuo e sincronizado de operações que permite otimizar o tempo de ciclo de cada atividade e, por consequência, a duração total de projeto. Referente aos fluxos de trabalho observou-se que o LPS tem ênfase na função controle do fluxo das equipes (ênfase nos planejamentos de médio e curto prazo). Entende-se que 
as potencialidades das diferentes abordagens de planejamento podem ser combinadas de forma a agregar as vantagens de cada sistema, de forma a obter os parâmetros prioritários estabelecidos para controle do sistema de produção.

Com o desenvolvimento futuro desta pesquisa, espera-se testar a hipótese de integração dos sistemas apresentada por meio de estudos de caso, como os apresentados nas referências, para verificar quais são as restrições e dificuldades existentes para sua implementação e quais os benefícios que poderão ser alcançados.

A contribuição esperada deste trabalho foi a revisão teórica sucinta de diferentes procedimentos de planejamento que podem se complementar e promover melhorias na gestão da produção, influenciando diretamente no desempenho global do empreendimento.

\section{AGRADECIMENTOS}

À Fundação de Amparo à Pesquisa do Estado de São Paulo (FAPESP), processo ${ }^{\circ}$ 2014/16362-1, pelo apoio na divulgação da pesquisa.

\section{REFERÊNCIAS}

ALVAREZ, R. R; ANTUNES JUNIOR, J. A. V. Takt-Time: conceitos e contextualização dentro do Sistema Toyota de Produção. Gestão \& Produção, v. 8, n. 1, p. 1-18, 2001.

BALLARD, G. et al. Production System Design in Construction. In: 9TH ANNUAL CONFERENCE OF THE INTERNATIONAL GROUP FOR LEAN CONSTRUCTION, 2001, Singapore. Proceedings... Singapore, 2001. p.1-15.

BALlARD, G. H. The Last Planner System of production control. 2000. 192p. Thesis (Doctoral degree in Philosophy) - Birmingham City University, Birmingham.

BERNARDES, M. M. S. Planejamento e controle da produção para empresas de construção civil. Rio de Janeiro, LTC, 2003. 190 p.

CHURCHMAN, C. W. "O que é um sistema?". In: Introdução à teoria dos sistemas. Tradução: Francisco M. Guimaraes. 2 ed. Petrópolis: Vozes, 1972. p.15-32.

FRANDSON, A; BERGHEDE, K; TOMMELEIN, I.D. Takt time planning for construction of exterior cladding. In: 21ST ANNUAL CONFERENCE OF THE INTERNATIONAL GROUP FOR LEAN CONSTRUCTION, Fortaleza, Brasil. Proceedings... Fortaleza, jul. 2013. p.1-10

GIL, A.C. Métodos e técnicas de pesquisa social. 6. ed. São Paulo: Atlas, 2012. 200 p.

KOSKELA, L. An exploration towards a production theory and its application to construction. 2000. 296 p. Thesis (Doctoral degree in Technology) - Technical Research Centre of Finland, Espoo.

MELLES, B; WAMELINK, J.W.F. Production control in construction. Delft, The Netherlands: Delft University Press, 1993.

OLIVEIRA, C. B. Avaliação de indicadores de planejamento e controle da produção na construção: boas práticas, eficácia e prazo. 2010. 194 f. Dissertação (Mestrado em Construção Civil) - Universidade Federal de São Carlos, São Carlos, 2010.

PULL PLANNING: lean construction. Produção de Jason Mayes. Dallas: The Beck Group, jan.2013. 1 vídeo (30 min), son., color. Disponível em: < http://youtu.be/5ecxE4XKrt4>. Acesso: maio, 2014.

SCHRAMM, F. K. O projeto do sistema de produção na gestão de empreendimentos habitacionais de interesse social. 2004. 182 p. Dissertação (Mestrado em Engenharia Civil) - Programa de PósGraduação em Engenharia Civil, Universidade Federal do Rio Grande do Sul, Porto Alegre.

SEPPANEN, O; BALLARD, G; PESONEN, S. The combination of last planner system and locationbased management system. Lean Construction Journal, Arlington, Virginia, EUA, p.15-34, 2010. 